\title{
Fighting Neuroblastomas with NBAT1
}

Editorial

\section{Gaurav Kumar Pandey and Chandrasekhar Kanduri}

Children are vulnerable to extracranial solid tumours, known as neuroblastomas, at a very young age. These tumors are derived from primitive sympathetic neural precursors and they account for almost $15 \%$ of all pediatric cancer deaths (1). The most distinguishing feature of these tumors is their heterogeneous clinical behavior, which can range from spontaneous regression to highly aggressive metastatic disease. This variability in the disease outcome depends on several factors such as the age of the patient, the stage of the disease, and the genetic profile.

In the last decade, we have witnessed major advances in the field of tumor biology and in drug development for treatment of various cancers including neuroblastoma. Despite these recent diagnostic and clinical advancements, the survival rate of patients with high-risk neuroblastomas still remains less than 50 per cent (2). The poor clinical outcomes in high-risk patients compel us to improve our understanding of these tumors further and to use more innovative approaches to identify candidate genes that may contribute to the disease.

Our recent study published in Cancer Cell journal has employed one such approach, whereby transcriptomes of low- and high-risk neuroblastomas were sequenced to identify differentially expressed long noncoding RNAs (lncRNAs) with possible roles in disease pathogenesis (3). LncRNAs are untranslated transcripts more than 200bp in size, with known regulatory functions in various key biological processes that have been implicated in development and differentiation (4). We identified a set of 24 unannotated lncRNAs as being significantly differentially expressed in low- and high-risk neuroblastomas. In addition, we also identified differentially expressed lncRNAs that mapped to characteristic non-random chromosomal aberrations associated with high-risk neuroblastomas such as the MYCN amplified region on chromosome 2 , and chromosomal deletions at $1 \mathrm{p}$ and $11 \mathrm{q}$ regions. These apparently differentially expressed lncRNA signatures might serve as biomarkers for determination of the prognosis of neuroblastomas, and their functional characterization would give major clues about the underlying biology of neuroblastoma pathogenesis.

One of the most interesting findings in our transcriptome study was the functional characterization of a lncRNA LOC729177 (known as neuroblastoma associated transcript1 or $N B A T 1$; formerly referred to as NBAT-1) that maps to a neuroblastoma hotspot on the 6 p22 region. This region has been shown to harbor three neuroblastoma associated SNPs and one of them-rs6939340-maps to intron 2 of NBAT1 (5). This IncRNA has specific clinical and functional characteristics that make it a highly interesting molecule regarding neuroblastoma. NBAT1 expression analysis in large datasets of 591 tumors showed that low expression is associated with poor clinical outcomes. Moreover, NBAT1 expression can be used as an independent prognostic marker for prediction of event-free survival in neuroblastoma patients. Our study demonstrated that the lower levels of NBAT1 in high-risk patients are due to two intricate transcriptional regulatory mechanisms involving $\mathrm{CpG}$ methylation and genotype at the high-risk neuroblastoma-associated SNP rs6939340. Hypermethylation of the NBAT1 promoter and $\mathrm{G} / \mathrm{G}$ genotype at the high-risk neuroblastoma-associated SNP were found to be significantly associated with lower

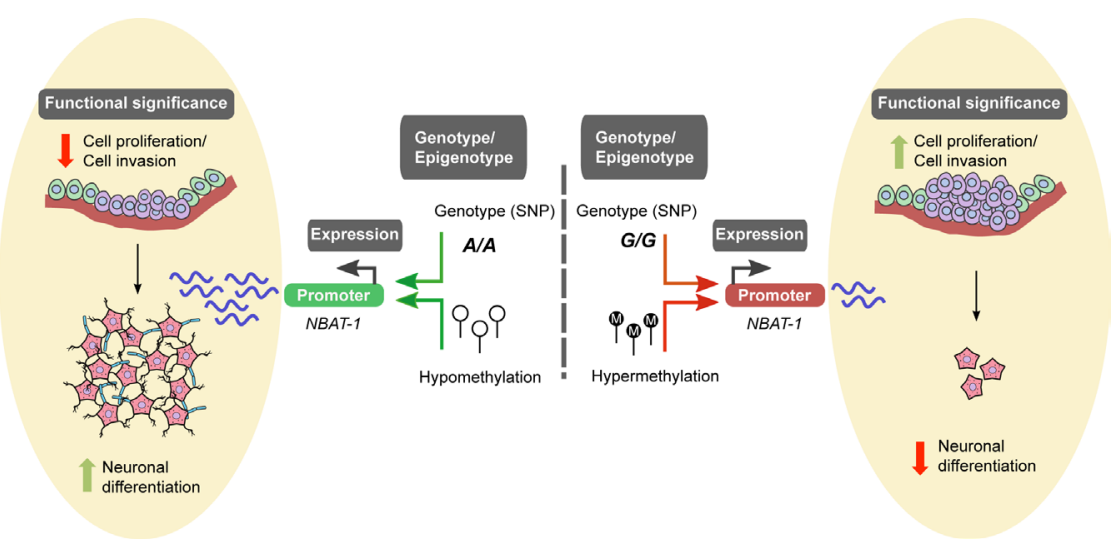

Figure: Model explaining functional role of $N B A T 1$ during neuroblastoma pathogenesis. 
expression of NBAT1 in high-risk patients, suggesting that both $\mathrm{CpG}$ methylation and $\mathrm{G} / \mathrm{G}$ genotype contribute to lower expression of $N B A T 1$ in high-risk patients.

Functional characterization of NBAT1 as a novel biomarker for neuroblastoma revealed its property as a tumor suppressor, making it a highly attractive target for novel therapeutic strategies for high-risk neuroblastoma patients. NBAT1 regulates biological processes that are highly critical for tumor development, such as cell proliferation and invasion by associating with PRC2 member, EZH2. This molecular interaction suppresses critical oncogenic networks, comprising the $S O X 9$, $O S M R$, and $V C A N$ genes, and affects the proliferative and invasive properties of neuroblastoma cells. The role of $N B A T 1$ as an effective tumor suppressor of neurobastomas complements its very important functions during neural lineage differentiation. Impaired neuronal differentiation of sympathetic neural precursors is one of the important features of high-risk neuroblastomas (6). NBAT1 expression is upregulated during retinoic acid induced neuronal differentiation of neuroblastoma cells. Its elevated levels suppress the negative regulator of the neuronal differentiation program $N R S F / R E S T$, thus promoting proper differentiation of neural precursor cells. Based on these observations, we proposed that a higher level of NBAT1 in low-risk tumors controls neuroblastoma progression and promotes neural differentiation. On the contrary, the hypermethylated NBAT1 promoter and the $\mathrm{G} / \mathrm{G}$ genotype at the disease associated SNP in high-risk neuroblastomas results in decreased expression of NBAT1, leading to highly proliferative and invasive neuroblastoma cells with an impaired ability to differentiation. This interesting correlation between lower expression of NBAT1 and poor differentiation in high-risk tumors makes it a hotspot for therapeutic intervention.

Our observations on NBAT1 open up novel lncRNA based treatment approaches for neuroblastomas. Furthermore, they affirm our hypothesis that lncRNAs are key molecules in determining the course of neuroblastoma disease. In conclusion, these observations have put IncRNA research in neuroblastoma disease under the spotlight and they have set the stage for further identification of novel lncRNA-based biomarkers for the risk stratification of neuroblastomas. They may also lead to druggable targets for neuroblastoma treatment.

Chandrasekhar Kanduri: Department of Medical Genetics, Institute of Biomedicine, Sahlgrenska Academy, University of Gothenburg, Gothenburg, Sweden

Correspondence: Chandrasekhar Kanduri, email Kanduri. Chandrasekhar@gu.se

Received: February 16, 2015;

Published: February 18, 2015;

\section{REFERENCES}

1. Brodeur GM. Nature reviews Cancer 2003; 3, 203-16.

2. Maris JM. The New England journal of medicine 2010; 362, 2202-11.

3. Pandey GK et al. Cancer cell 2014; 26, 722-37.

4. Whitehead J et al. Biochim Biophys Acta 2009; 1790, 93647.

5. Maris JM et al. The New England journal of medicine 2008;358(24):2585-93.

6. Nakagawara A et al. The New England journal of medicine 1993;328(12):847-5 